\title{
LOUVAIN NATURAL RADIOCARBON MEASUREMENTS VII
}

\author{
E. GILOT
}

Department of Nuclear Chemistry, University of Louvain,

Louvain, Belgium

The following list comprises selected measurements made during 1967-68. The method is essentially the same as previously described. A $0.6 \mathrm{~L}$ proportional gas-counter at $3 \mathrm{~atm} \mathrm{CH}_{4}$ pressure is used. Ages are given relative to A.D. 1950 and half-life of $5570 \mathrm{yr}$ has been assumed. The quoted error is the experimental standard deviation and includes the uncertainty on the unknown sample, the modern standard and the background.

The description of each sample is based on information supplied to the laboratory by the submitters.

Sincere thanks are due Prof. P. C. Capron for his constant guidance. Thanks are also due F. Frix for his help in operating the laboratory and to $G$. Michotte for maintenance of electronics. Financial support was provided by Institut Interuniversitaire des Sciences Nucléaires, Brussels.

\section{SAMPLE DESCRIPTIONS}

\section{Oorderen II series}

I. GEOLOGIC SAMPLES

Peat from Oorderen $\left(51^{\circ} 20^{\prime} \mathrm{N}\right.$ Lat, $4^{\circ} 19^{\prime} \mathrm{E}$ Long), Prov. of Antwerp, Belgium, alt $2 \mathrm{~m}$. Holocene peat layer, $1.50 \mathrm{~m}$ thick, covered by clay of the Polders. Coll. 1963, pollen analyzed and subm. by A. Munaut, Univ. of Louvain, Lab. of Palynology and Dendrochronology. Pollen diagram is to be compared with those from Terneuzen and Zandvliet (Munaut, 1967).

\section{Lv-248. Oorderen II.1}

$$
2220 \pm 70
$$

Peat from 19 to $26 \mathrm{~cm}$. Pollen diagram shows 2nd maximum of Fagus in beginning of Sub-Atlantic period. At Zandvliet a 2nd Sub-Atlantic maximum of Fagus is dated A.D. 940 (Lv-253, this list). Further investigations are necessary.

\section{Lv-249. Oorderen II.2}

Peat from 40 to $45 \mathrm{~cm}$. 1st Sub-Atlantic maximum of Fagus. Date agrees with those for same event at Terneuzen (Lv-117, Radiocarbon, 1964, v. 6, p. 163) and Zandvliet (Lv-254, this list).

\section{Lv-250. Oorderen II.3}

$$
3800 \pm 70
$$

Peat from 60 to $65 \mathrm{~cm}$. Just before Sub-Boreal decrease of Ulmus, not correlative with classical decrease. Same event is dated 1800 B.c. at Terneuzen (Lv-122, Radiocarbon, 1965, v. 7, p. 118) and 1840 B.c. at Zandvliet (Lv-255, this list). 
Lv-251. Oorderen II.4

ning of the Sub-Borat to $115 \mathrm{~cm}$. Pollen diagram shows at this level beginto Ir-116 (Radiocarbon, 1964 of Ulmus. Date a little too old according to $\mathrm{L} v-116$ (Radiocarbon, 1964, v. 6, p. 163), Lv-123 (Radiocarbon, 1965, v. 7, p. 119) and Lv-256 (this list).

\section{Zandvliet VIII series}

Peat from Zandvliet ( $51^{\circ} 21^{\prime} \mathrm{N}$ Lat, $4^{\circ} 17^{\prime} \mathrm{E}$ Long), Prov. of Antwerp, Belgium, alt $2 \mathrm{~m}$. Holocene peat layer, $3.5 \mathrm{~m}$ thick, under clay layer of the Polders. Coll. 1962, pollen analyzed and subm. by A. Munaut. Pollen diagram is correlated with those from Terneuzen and Oorderen (Munaut, 1967).

\section{Lv-253. Zandvliet VIII.1}

$1010 \pm 90$

Peat from 50 to $55 \mathrm{~cm}$. Pollen analysis indicates 2nd Sub-Atlantic maximum of Fagus.

\section{Lv-254. Zandvliet VIII.2}

$2350 \pm 110$

\section{B.C.} 320 Peat from 100 to $105 \mathrm{~cm}$, 1st Sub-Atlantic maximum of Fagus, dated 320 B.C. at Terneuzen (Lv-117, Radiocarbon, 1964, v. 6, p. 163) and 440 13.C. at Oorderen (Lv-249, this list).

\section{Lv-255. Zandvliet VIII.3}

$3790 \pm 160$

1840 B.C. firms Lv-122 (Radiocarbon, 1965, v. 7, p. 118) and Lv-250 (this list).

\section{Lv-256. Zandvliet VIII.4}

$4480 \pm 110$

Peat from 235 to $240 \mathrm{~cm}$. Pollen diagram shows Atlantic-Sub-Boreal transition, with the last increase of Ulmus. At Terneuzen, this transition is situated between 2330 B.C. (Lv-116, Radiocarbon, 1964, v. 6, p. 163) and 2640 в.c. (Lv-123, Radiocarbon, 1965, v. 7, p. 119).

\section{Lv-260. Solwaster}

$2310 \pm 100$

Wood from peat bog at Solwaster $\left(50^{\circ} 31^{\prime} \mathrm{N}\right.$ 360 B.c.

Sart lez Spa, Prov. of Liege, Belgium, . Found at $0.60 \mathrm{~m}$ depth by J. M. Deumer, Spa Monopole Soc.

\section{ARCHAEOLOGIC SAMPLES}

\section{Pincevent series, France}

Charcoal sediment from fabricated hearth of open-air prehistoric

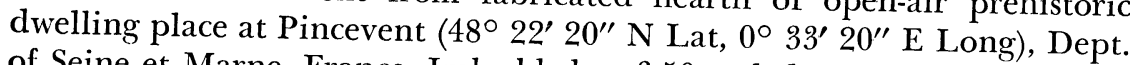
of Seine et Marne, France. Imbedded at $3.50 \mathrm{~m}$ below ground surface in clay horizon, $2 \mathrm{~m}$ thick, overlain by sand layer. At same level, Late Mag- 
dalenian industry (Leroi-Gourhan and Brezillon, 1967). Coll. 1964 and subm. by A. Leroi-Gourhan, Centre de Recherches Préhistoriques et Protohistoriques, Univ. of Paris (Sorbonne).

General Comment: samples are not pretreated. Organic matter, finely divided and adsorbed on clay, was chemically extracted by solubilization in $\mathrm{NaOH}$ and precipitation by $\mathrm{HCl}$. Ages are thus minimum, because possible recent humic contamination is not removed. However, C-14 ages agree with archaeol.

\section{Lv-291. Pincevent 1}

Upper level of Hearth I.

\section{Lv-292. Pincevent 2}

Lower level of Hearth I.

\section{Lv-293. Pincevent 3}

$10,920 \pm 540$ 8970 B.C.

\section{$11,610 \pm 400$}

9660 B.c.

$11,310 \pm 330$

\section{Kinshasa series, Congo}

Series dates prehistoric industries in Kinshasa plain (Van Moorsel, 1968). Parts are publ. in Radiocarbon: 1962, v. 4, p. 95; 1964, v. 6, p. 160; 1965 , v. 7, p 118. All dates agree with each other and with chronology of industries. Coll. 1965 by H. Van Moorsel, Prehistorical Mus. Lovanium Univ., Kinshasa; subm. by F. Gullentops, Univ. of Louvain.

\section{Lv-287. Basoko}

$12,230 \pm 250$ 10,280 в.c.

Charcoal from valley of Basoko R. (4 $4^{\circ} 21^{\prime} \mathrm{S}$ Lat, $15^{\circ} 17^{\prime} \mathrm{E}$ Long), Prov. of Kinshasa, Congo. Charcoal imbedded at ca. $1.5 \mathrm{~m}$ depth in muddy yellow sand $3 \mathrm{~m}$ thick overlying polymorphous sandstone blocks. Sample related to evolving Middle Lupembian stone industry. Just above sandstone layer, Older Lupembian industry dated > 26,000 (Lv-163, Radiocarbon, 1965 , v. 7, p. 121). In clay bank of river, Tshitolian horizon dated $9730 \pm 200$ B.P. (Lv-164, Radiocarbon, 1965, v. 7, p. 121). Date agrees with other Middle Lupembian site at Cabu (Lv-166, Radiocarbon, 1965 , v. 7 , p. $121: 15,080 \pm 480$ в.P.)

\section{Lv-288. Kinsuka}

$3870 \pm 90$

1920 B.c.

Charcoal from Kinsuka (4 $20^{\prime} 38^{\prime \prime}$ S Lat, $15^{\circ} 12^{\prime} 45^{\prime \prime}$ E Long), Prov. of Kinshasa, Congo. Imbedded at $2 \mathrm{~m}$ depth in 5-m-thick alluvion bank of Congo R. Nearby the top, Late Tshitolian remains. At base on red sandstone, a gravel layer with altered stone industry estimated Middle Lupembian. Sample, not directly related to prehistoric industry, gives minimum age to alluvions. 


\section{Lv-289. Kizenzu}

Charcoal from Kizenzu (4 $24^{\prime} 51^{\prime \prime}$ S Lat, $15^{\circ} 19^{\prime} 51^{\prime \prime}$ E Long), Prov. of Kinshasa, Congo. From $60 \mathrm{~cm}$ depth in marshy white sand. At same level, Late Tshitolian industry and old potsherds. Date agrees with other Late Tshitolian site at Gafula (Lv-45, Radiocarbon, 1962, v. 4, p. 97 , and Lv-162, Radiocarbon, 1965 , v. 7 , p. 121) dated $5830 \pm 180$ в.C. and 5750 \pm 110 B.c. Potsherds probably come from intrusive pottery; stratigraphy gives no evidence.

\section{Sampont series}

Wood substructure from Sampont $\left(49^{\circ} 40^{\prime} 50^{\prime \prime} \mathrm{N}\right.$ Lat, $6^{\circ} 09^{\prime} 55^{\prime \prime} \mathrm{E}$ Long), Prov. of Luxembourg, Belgium. Squared beams found at $1.40 \mathrm{~m}$ depth under several alluvion layers. In same site, 6 square piles, $1.50 \mathrm{~m}$ long, vertically driven in ground. Coll. 1966 and subm. by J. Noel. Attributed to road joining Roman road from Reims to Treves across "Marais de Vance" (Noel, 1967). Date also gives information about gravelly alluvions of the Semois R. (Coûteaux, 1967).

\section{Lv-345. Sampont 1966 B}

Aller beam (Alnus, id. by J. Heim), in situ.

Lv-346. Sampont $1966 \mathrm{C}$

Oak beam, exhumed in 1963.

\section{Lv-347. Sampont 1966 D}

From same beam as Lv-346.

$$
250 \pm 100
$$

A.D. 1700

$$
\begin{array}{r}
500 \\
\text { A.D. } 1450
\end{array}
$$

$$
730 \pm 90
$$

A.D. 1220

$$
4040 \pm 90
$$

2090 B.C.

\section{Lv-290. Chaumont-Gistoux}

Charcoal from Chaumont-Gistoux $\left(50^{\circ} 41^{\prime} \mathrm{N}\right.$ Lat, $4^{\circ} 42^{\prime} \mathrm{E}$ Long), Prov. of Brabant, Belgium. Found $1.10 \mathrm{~m}$ below ground surface, mixed with Michelsberg-type potsherds. Coll. 1965 by Y. Graff; subm. by A. Munaut. Charcoal is related to pollen diagram by A. Munaut, showing at this level Atlantic-Sub-Boreal transition. C-14 date agrees with archaeol. and palynological estimations.

\section{Lv-301. Vaux sous Chèvremont}

$$
1040 \pm 80
$$

Charcoal from feudal castle of Chèvremont $\left(50^{\circ} 36^{\prime} \mathrm{N}\right.$ Lat, $5^{\circ} 37^{\prime} \mathrm{E}$ Long) at Vaux sous Chèvremont, Prov. of Liège, Belgium, alt $196 \mathrm{~m}$. Sample from burned horizon in Excavation V, 66 VC 23, at $1.30 \mathrm{~m}$ depth. Coll. 1966 by Papeleux; subm. by J. Mertens, Univ. of Louvain, Archaeol. Inst. Castle was destroyed in 10th century; C-14 date agrees with archaeol. Another sample from same site (Lv-228, Radiocarbon, 1966, v. 8, 
p. 254) is dated $540 \pm 80$ B.P., probably because of presence of many recent rootlets.

\section{Abbaye des Dunes series}

Samples from Abbaye des Dunes at Koksijde $\left(51^{\circ} 06^{\prime} 20^{\prime \prime}\right.$ N Lat, $6^{\circ}$ 05' 35" E Long), Prov. of W Flanders, Belgium, alt $10 \mathrm{~m}$. Site was certainly occupied as early as High Middle ages. Abbey was definitively forsaken in A.D. 1579. Now archaeol. layer is overlain by $7 \mathrm{~m}$ sand. Coll. 1965-66 by P. Schittekat, Abbaye des Dunes; subm. by M. Parmentier, Univ. of Louvain.

Lv-340. Abbaye des Dunes, Sample 2

Charred wood found at surface of archaeol. layer in cess-pit contemporaneous with abbey. Several glass articles were also found in pit (Parmentier, 1968).

\section{Lv-341. Abbaye des Dunes, Sample 3}

$350 \pm 120$

Wood from pale driven $1 \mathrm{~m}$ deep at $\mathrm{N}$ side of church.

Lv-342. Abbaye des Dunes, Sample 3 bis

Collagen from bone found with Lv-341.
A.D. 1490

$$
460 \pm 100
$$

\section{Lv-343. Abbaye des Dunes, Sample 4}

$520 \pm 120$

Wood from humic layer, $1 \mathrm{~m}$ deep, date of which is assumed end of 16 th century.

\section{Lv-348. Abbaye des Dunes, Sample 1}

$870 \pm \mathbf{1 4 0}$

Wood from geogr. center of necropolis of High Middle ages at $2 \mathrm{~m}$ below archaeol. level. Central part of necropolis was again handled probably during beginning of 12 th century. Date agrees with this assumption (Schittekat, 1968).

\section{REFERENCES}

Date lists:

Louvain I

Louvain II

Louvain III

Louvain IV

Dossin, Deumer, Capron, 1962

Deumer, Gilot, Capron, 1964

Gilot, Ancion, Capron, 1965

Gilot, Ancion, Capron, 1966

Coûteaux, M., 1967, Evolution du paysage végétal du Bas-Luxembourg depuis le Pléni-Würm: thesis, Bot. Inst. Univ. Louvain, $413 \mathrm{p}$

Deumer, J. M., Dossin, J. M., Capron, P. C.,
measurements I: Radiocarbon, v. 4, p. 95-99.

Deumer, J. M., Gilot, E., Capron, P. C., 1964, Louvain natural radiocarbon measurements II: Radiocarbon, v. 6, p. 160-166.

Gilot, E., Ancion, N., Capron, P. C., 1965, Louvain natural radiocarbon measurements III: Radiocarbon, v. 7, p. 118-122.

1966, Louvain natural radiocarbon measurements IV: Radiocarbon, v. 8 , p. $248-255$. 
Leroi-Gourhan, A. and Brezillon, M., 1967, L'habitation magdalénienne $\mathrm{n}^{\circ} 1$ de Pincevent: Gallia-Préhistoire, v. 9, fasc. 2, 1966, p. 263-385.

Munaut, A., 1967, Recherches paléo-écologiques en Basse et Moyenne Belgique: Acta Geographica Lovaniensia, v. 6, 191 p.

Noel, J., 1967, Découverte archéologique dans la vallée de la Semois à Sampont: Inst. Archéol. du Luxembourg Bull., no. 1-2, p. 39-45.

Parmentier, M., 1968a, Un "Krautstrunk" à Koksijde: Centre scientif. et culturel de l'Abbaye des Dunes et du Westhoek Bull., no. 11, p. 120-130.

1968b, La datation par loek Bull, no. 11, p. 120-130. des Dunes et du Westhoek Bull., no. 11, p. 172-175.

Schittekat, P., 1968, Compte-rendu des fouilles 1966-67: de l'Abbaye des Dunes et du Westhoek Bull,

kull., no. 11, p. 68-79.

Plehistoire de la Plaine de Kinshasa: Lovanium Univ. of Kinshasa Ed., 287 p. 